\title{
Multicentered solution for maximally charged dilaton black holes in arbitrary dimensions
}

\author{
Kiyoshi Shiraishi \\ Akita Junior College, Shimokitade-Sakura, Akita-shi, Akita 010, Japan
}

J. Math. Phys. 34, 1480-1486 (1993)

\begin{abstract}
A family of static multicentered solutions to modified Einstein-Maxwell equations coupled with a dilaton is constructed in $(1+N)$ dimensional space-time $(N \geq 2)$. For $N \geq 3$, the solutions are generalizations of the Majumdar-Papapetrou solution. We also find the solution in $(1+2)$ dimensions, where the scalar and vector forces cancel each other in the static case. The interaction between two extreme charged dilaton black holes in the low-energy limit is investigated in $(1+N)$ dimensions $(N \geq 3)$. We find that there remains the residual velocity-dependent force in general cases, except for the case with $N=a^{2}$.
\end{abstract}

\section{INTRODUCTION}

There has recently been a revived interest in the exact solutions in the coupled system with a dilaton. [1, 2, 3, 4] Although the study of dilaton-coupled Einstein equations has been motivated by string theory and Kaluza-Klein theory, it has recently turned out that black holes described by the solution for the generalized dilaton coupling have curious properties.[1, 2, 3, 4, 5]

The multi-black-hole solution in usual Einstein-Maxwell system is known as the Papapetrou-Majumdar metric,[6] which represents the static equilibrium among extreme Reissner-Nordstrom black holes. Recently, the multicentered solution in string theory has been found in Ref. [2]. The difference between these two solutions originates from the coupling to the dilaton in the case of string theory. Actually it has been suggested that the extreme- black-hole solution in string theory has peculiar nature.[2]

We thus come to take an interest in interpolating the two cases: In this paper, we consider the generalized dilaton coupling in modified Einstein-Maxwell system. We construct the multi-black-hole solutions in various dimensions. The generalized Papapetrou-Majumdar solutions in $(1+N)$ dimensional EinsteinMaxwell system without the dilaton have been discussed by Myers. [7] The solutions we obtain in Sec. 2 contains Myers' solution as a solution in the limit of dilaton decoupling. 
Further, we show the static multicentered solution in $(1+2)$ dimensions in Sec. 3 .

In Sec. 4, we study the interaction between two maximally charged dilaton black holes in the low-energy limit. The last section is devoted to conclusion.

\section{THE MULTIDILATON BLACK-HOLE SO- LUTION IN FOUR AND MORE THAN FOUR DIMENSIONS}

We start with the action

$$
S=\int d^{N+1} x \frac{\sqrt{-g}}{16 \pi}\left[R-\frac{4(\nabla \phi)^{2}}{N-1}-e^{-\{4 a /(N-1)\} \phi} F^{2}\right],
$$

where we set the Newton constant equals to one. The constant $a$ (which can be taken as non-negative) is the parameter which determines the strength of the coupling between the Maxwell field $F$ and the dilaton field $\phi$. For $a=0$, the action describes the usual Einstein-Maxwell system with a free scalar field: a charged nonrotating black hole in the system is represented by the ReissnerNordstrom solution. For $a=1$, the action reduces to the one which derived from the low-energy string theory.

For $N=3, a=0$ case, multicentered static solutions to the Einstein-Maxwell equations were found by Papapetrou and Majumdar.[6] Further, generalization to higher dimensions was attained by Myers.[7] Their solutions correspond to a many-body system of extreme charged black holes, where the gravitational attraction and the Coulomb repulsion is exactly canceled. In the present paper, we manage to extend their studies to the system including the dilaton field. As a result, the scalar force between black holes come to take part in the system.

In this section, we treat the case with $N \geq 3$. We handle the $N=2$ case separately in the later section, because the solution in $(1+2)$ dimensions behaves very differently from the ones in higher dimensions.

The extremity condition for charged dilaton black holes in arbitrary dimensions can be read from the results in Refs. [1]. If we fix the asymptotic value of the dilaton at spatial infinity to be zero, we flnd that the extremity condition is expressed as the following ratio of charge to mass:

$$
\frac{|Q|}{m}=\frac{8 \pi}{A_{N-1}}\left(\frac{N-2+a^{2}}{2(N-1)}\right)^{1 / 2},
$$

where $A_{N-1}=2 \pi^{N / 2} / \Gamma(N / 2)$. Please note that the expression would take a different form in accordance with the choice of the normalization of the charge. The present notation coincides with Ref. [2] when $N=3$ and $a=1$. The extremity condition must be realized for each point "source" in the multi-blackhole solution. The extremity and balance condition will be con- sidered further in Sec. 4. 
Now we turn to the multicentered solution. The general metric ansatz (for $N \geq 3$ ) can be written in the isotropic coordinates:[7]

$$
d s^{2}=-U^{-2}\left(\mathbf{x}^{k}\right) d t^{2}+U^{2 /(N-2)}\left(\mathbf{x}^{k}\right) \delta_{i j} d \mathbf{x}^{i} d \mathbf{x}^{j} .
$$

We also assume that the electric potential and the dilaton configuration are proportional to some powers of $U\left(\mathrm{x}^{k}\right)$. Solving the equations of motion obtained from the action (1) we get the general form of $U\left(\mathbf{x}^{k}\right)$ for an " $n$-body" system:

$$
U\left(\mathbf{x}^{k}\right)=\left\{F\left(\mathbf{x}^{k}\right)\right\}^{(N-2) /\left(N-2+a^{2}\right)},
$$

where

$$
F\left(\mathbf{x}^{k}\right)=1+\frac{1}{N-2} \sum_{i=1}^{n} \frac{\mu_{i}}{\left|\mathbf{x}-\mathbf{x}^{i}\right|^{N-2}},
$$

and using this expression, we obtain the potential and the dilaton configuration:

$$
A= \pm\left(\frac{N-1}{2\left(N-2+a^{2}\right)}\right)^{1 / 2}\left\{F\left(\mathbf{x}^{k}\right)\right\}^{-1} d t
$$

and

$$
e^{-\{4 a /(N-1)\} \phi}=\left\{F\left(\mathbf{x}^{k}\right)\right\}^{2 a^{2} /\left(N-2+a^{2}\right)} .
$$

The constant $\mu_{i}$ has the following connection to the mass and the charge of each source:

$$
\begin{gathered}
m_{i}=\frac{A_{N-1}(N-1)}{8 \pi\left(N-2+a^{2}\right)} \mu_{i}, \\
\left|Q_{i}\right|=\left(\frac{N-1}{2\left(N-2+a^{2}\right)}\right)^{1 / 2} \mu_{i} .
\end{gathered}
$$

In this solution, we have fixed the asymptotic value for $\phi$ to be zero.

The solution obtained here coincides with the solution of Myers[7] in the $(1+N)$-dimensional Einstein-Maxwell system when $a=0$; therefore, of course, our solution includes the Papapetrou-Majumdar solution[6] as a special case for $\mathrm{N}=3$. The multi-black-hole solution shown by the authors of Ref. [2] can be rederived if $N=3$ and $a=1$ are substituted in the present solution.

The discussion on the physical interpretation of the solution is left for the subject of Sec. 4.

\section{CHARGED DILATON SOLITON IN $(1+2)-$ DIMENSIONAL SPACE-TIME}

In three-dimensional space-time, there is no propagating graviton mode in Einstein gravity; nevertheless, the global geometry of space-time is governed by Einstein equations. Various aspects of three-dimensional Einstein gravity have been investigated by many authors in the past decade[8, 9] (and see also Ref. [10]). 
In three dimensions, there is no black-hole solution; thus the extreme charged object in $(1+2)$ dimension seems to make no sense. The static, charged manybody system cannot exist because there is no attractive gravitational force to balance with the Coulomb force.

There is another story in the Einstein-Maxwell dilaton system. The scalar force induced by the dilaton can cancel the electric force. Another prospect is obtained from the extremity condition. If the condition (2) can be extrapolated to the $N=2$ case, it shows $|Q| / m=2^{3 / 2} a$. Thus in the absence of the dilaton, maximal charge of a "charged source" is found to be zero. This interpretation sounds reasonable; the metric for the static configuration of an arbitrary number of point masses are known in the three-dimensional Einstein gravity.[8] This corresponds to the three-dimensional extension of the Papapetrou-Majumdar solution.

Now we first get the "spherical" solution in the three-dimensional EinsteinMaxwell dilaton system. The action is given by (1) with $N=2$ substituted. Using the isotropic coordi- nates, one can find the exact solution

$$
d s^{2}=-d t^{2}+\left|\mathbf{x}^{2}\right|^{-4 m}|f(\mathbf{x})|^{2 / a^{2}} \delta_{i j} d \mathbf{x}^{i} d \mathbf{x}^{j},
$$

with

$$
\begin{gathered}
e^{-2 a \phi}=f(\mathbf{x})=-C \ln \left(|\mathbf{x}| / r_{c}\right), \\
e^{-4 a \phi} F=\frac{Q}{|\mathbf{x}|} \frac{\mathbf{x}^{i}}{|\mathbf{x}|} d t \wedge d \mathbf{x}^{i},
\end{gathered}
$$

where $m, C$, and $r_{c}$ are constants. Note that the solution has singularities at a finite distance $r$, besides the origin. The present solution has no smooth connection to the $a=0$ case. In Ref. [9], the Einstein-Maxwell equations is solved in three dimensions. Their solution also has the singularity at a finite distance.

More notable remark is on $m$, which can take any value in general. The total mass of the object described by the solution is not well defined because of the divergence of the energy of the electric field. Thus we cannot call $m$ as mass in a precise sense. At the same time, in three dimensions, the asymptotic flat condition cannot be attained in a practical sense. This is crucial for studying the properties of any solution to the three-dimensional Einstein equation.

The multicentered solution can also be obtained in the isotropic coordinates. We find that the solution is given by

$$
d s^{2}=-d t^{2}+G(\mathbf{x})|f(\mathbf{x})|^{2 / a^{2}} \delta_{i j} d \mathbf{x}^{i} d \mathbf{x}^{j},
$$

with

$$
\begin{gathered}
e^{-2 a \phi}=f(\mathbf{x})=-2^{1 / 2} a \sum_{i=1}^{n} Q_{i} \ln \left(\left|\mathbf{x}-\mathbf{x}_{i}\right| / r_{c i}\right), \\
e^{-4 a \phi} F=\sum_{i=1}^{n} \frac{Q}{|\mathbf{x}|} \frac{\mathbf{x}^{i}}{|\mathbf{x}|} d t \wedge d \mathbf{x}^{i}
\end{gathered}
$$


and

$$
G(\mathbf{x})=\prod_{i=1}^{n}\left(\left|\mathbf{x}-\mathbf{x}_{i}\right| / b_{i}\right)^{-8 m_{i}} .
$$

Here $m_{i}$ (and $b_{i}$ ) can take arbitrary values. Actually, $\ln G$ is nothing but the solution to the two-dimensional Laplace equation. The ambiguous situation come from the fact that we cannot constrain the behavior of the metric at asymptotic region of space in $(1+2)$ dimensions.

The naive extension of the extremity condition turns out wrong. We can, however, find an interesting similarity to the solution for the other dimensions. To carry out the analysis, we must set $G(x)=1$ in the solution (13). This could be fulfilled by imposing an appropriate condition near the point charge, instead of the asymptotic behavior.

The idea is to look into the vicinity where $f \sim 1$ around a point source. In that place, the metric (13) of space behave as

$$
g_{i j} \sim\left\{1-\left(2^{3 / 2} / a\right) Q_{i} \ln \left(\left|\mathbf{x}-\mathbf{x}_{i}\right| / r_{c}^{\prime}\right)\right\} \delta_{i j},
$$

where $r_{c}^{\prime}$ is a constant. On the other hand, we consider a point mass mi. The space component of the metric is approximately given in the vicinity of unity:

$$
g_{i j}(\text { point mass })=\left(\left|\mathbf{x}-\mathbf{x}_{i}\right| / b_{i}\right)^{-8 m_{i}} \delta_{i j} \sim\left\{1-8 m_{i} \ln \left(\left|\mathbf{x}-\mathbf{x}_{i}\right| / b_{i}\right)\right\} \delta_{i j}
$$

If we try to identify the behaviors, we must take

$$
Q_{i} / m_{i}=2^{3 / 2} a .
$$

This is no more than the "naively" extended extremity condition! In higher dimensions, the asymptotic region corresponds to the space component of the metric $g_{i j} \sim \delta_{i j}$.

The further implication of the solution will be exhibited in Sec. 4 .

\section{THE INTERACTION BETWEEN TWO MAX- IMALLY CHARGED DILATON BLACK HOLES IN ARBITRARY DIMENSIONS}

First we examine the balance condition in a physical perspective. The system we have treated is governed by three kinds of forces; the Newtonian attraction, the Coulomb repulsion, and the attractive scalar force mediated by the dilaton. We can define the scalar charge $\sigma$ by the asymptotic behavior of the dilaton as

$$
\frac{2^{1 / 2}}{(N-1)^{1 / 2}} \nabla_{i} \phi \sim-\frac{\sigma}{|\mathbf{x}|^{N-1}} \frac{\mathbf{x}^{i}}{|\mathbf{x}|} \quad \text { as } \quad|\mathbf{x}| \rightarrow \infty,
$$

because of the normalization required from the realization of the "proper" kinetic term (note, however, the normalization in Ref. [1] is different from ours; 
this is due to the difference in the normalization of the kinetic term for the vector field). The normalization here is chosen for the correspondence to the Coulomb potential.

The Newtonian force is given by

$$
\left(F_{N}\right)_{i}=-m \nabla_{i} \phi_{N}
$$

where the Newton potential $\phi_{N}$ is defined via

$$
g_{00} \sim-\left(1+2 \phi_{N}\right) \text { as }|\mathbf{x}| \rightarrow \infty,
$$

as the leading term.

Consequently, the amount of the total force between two point sources $a$ and $b$ separated in the distance is written as

$$
\frac{A_{N-1}}{4 \pi}\left[\frac{Q_{a} Q_{b}}{R_{a b}^{N-1}}-\frac{\sigma_{a} \sigma_{b}}{R_{a b}^{N-1}}-\left(\frac{4 \pi}{A_{N-1}}\right)^{2} \frac{2(N-2)}{N-1} \frac{m_{a} m_{b}}{R_{a b}^{N-1}}\right],
$$

where $R_{a b}$ is the distance between the point sources $a$ and $b$. The unpleasant factor $A_{N-1} / 4 \pi$ appears in our convention, since we must sweep the dependence on the space-time dimension into somewhere. One can immediately confirm the fact that the total force (23) vanishes in the system described by the static solution in Sec. 2, substituting the relations (8) and (9) and taking (20) into account.

We can also see the balance between the electric and the scalar forces in $(1+2)$ dimensions. In this case, we evaluate the forces at $f\left(\mathbf{x}^{k}\right) \sim 1$, i.e., $g_{i j} \sim \delta_{i j}$.

The consideration here is to show the physical implication; it should be recognized that the static balance among the forces holds at any distance in the exact solutions in any dimensions.

Next let us consider the slow motion of the point sources. Recently the slow motion of classical lumps or solitons in many kind of field theoretical models has been analyzed.[11, 12, 13, 14, 15, 16] The low-energy scattering of extreme Reissner-Nordstrom black holes has been studied in Refs. [15] and [16]. It seems very useful to introduce the effective Lagrangian in the low-energy limit in gravitating systems.[14, 15, 16, 17] We wish to find the effective Lagrangian for the system which consists of two maximally charged dilaton black holes in $(1+N)$ dimensions $(N \geq 3)$. The generalization to the many-body system would be a straightforword task.

Here the low-energy limit means the situation where any radiation reaction can be ignored. Practically this means, in the effective Lagrangian, keeping terms up to quadratic in the relative velocities of the constituents of the system.

We must be careful not to take double counting of the contribution of different interactions. After some straightforword calculation, we obtain an effective Lagrangian for two point sources (where the velocity of light $c$ is explicitly indicated):

$$
L=\frac{1}{2} m_{a} v_{a}^{2}\left(1+\frac{v_{a}^{2}}{4 c^{2}}\right)+\frac{1}{2} m_{b} v_{b}^{2}\left(1+\frac{v_{b}^{2}}{4 c^{2}}\right)
$$




$$
\begin{aligned}
& +\frac{A_{N-1}}{4 \pi(N-2) R_{a b}^{N-2}}\left[Q_{a} Q_{b}-\sigma_{a} \sigma_{b}-\left(\frac{4 \pi}{A_{N-1}}\right)^{2} \frac{2(N-2)}{N-1} m_{a} m_{b}\right] \\
& +\frac{A_{N-1}\left(v_{a}^{2}+v_{b}^{2}\right)}{8 \pi c^{2}(N-2) R_{a b}^{N-2}}\left[\left(\frac{4 \pi}{A_{N-1}}\right)^{2} \frac{2 N}{N-1} m_{a} m_{b}-\sigma_{a} \sigma_{b}\right] \\
& +\frac{A_{N-1}\left(\mathbf{v}_{a} \cdot \mathbf{v}_{b}\right)}{8 \pi c^{2}(N-2) R_{a b}^{N-2}}\left[Q_{a} Q_{b}+\sigma_{a} \sigma_{b}-\left(\frac{4 \pi}{A_{N-1}}\right)^{2} \frac{2(3 N-2)}{N-1} m_{a} m_{b}\right] \\
& +\frac{A_{N-1}\left(\mathbf{n} \cdot \mathbf{v}_{a}\right)\left(\mathbf{n} \cdot \mathbf{v}_{b}\right)}{8 \pi c^{2} R_{a b}^{N-2}}\left[Q_{a} Q_{b}-\sigma_{a} \sigma_{b}-\left(\frac{4 \pi}{A_{N-1}}\right)^{2} \frac{2(N-2)}{N-1} m_{a} m_{b}\right] \\
& +O\left(1 / R_{a b}^{N-1}\right),
\end{aligned}
$$

where $\mathbf{n}$ is the unit vector in the direction $b-a$. For the static configuration, we have found (for $i=a, b$ )

$$
\begin{gathered}
m_{i}=\frac{A_{N-1}(N-1)}{8 \pi\left(N-2+a^{2}\right)} \mu_{i}, \\
\left|Q_{i}\right|=\left(\frac{N-1}{2\left(N-2+a^{2}\right)}\right)^{1 / 2} \mu_{i}, \\
\left|\sigma_{i}\right|=a\left(\frac{N-1}{2}\right)^{1 / 2} \frac{1}{N-2+a^{2}} \mu_{i} .
\end{gathered}
$$

When (25), (26), and (27) are substituted, the second line of (4.5) vanishes trivially. This means no more than the balance condition.

Further going on examining when each term in (24) vanishes, we encounter a simple result. The third and fourth lines of $(24)$ vanish when $a^{2}=N$. The fifth line vanishes identically, regardless of the value of $a$. Thus all the interactions (of the order up to $v^{2}$ ) disappear if and only if $N=a^{2}$. The case for $N=3$ can be related to the Kaluza-Klein monopole[14] through the duality. To analyze this case closely is beyond the scope of the present paper and will be reported in a separate publication.

Since the solution is a unique solution, point particles in general EinsteinMaxwell dilaton systems feel the residual velocity-dependent forces mutually.

\section{CONCLUSION}

We have found $(1+N)$-dimensional, static, multicentered solutions to the Einstein-Maxwell equations coupled with dilaton. The solutions stand for static configurations of many-body systems of maximally charged dilaton black holes $(N \geq 3)$.

We have also found that there is no interaction up to quadratic in $v / c$ between two extreme charged dilaton black holes if and only if $N=a^{2}$. This case 
is related to the Kaluza-Klein monopole $(N=3)$ (Ref. [14]) via the duality in the electromagnetism in four dimensions.

We wish to report the thorough investigation of the slow motion of the extreme dilaton black holes in the future. We are also interested in the study of

the connection between the solutions obtained in this paper and supersymmetric models in diverse dimensions.

\section{References}

[1] G. W. Gibbons and K. Maeda, Nucl. Phys. B298, 741 (1988).

[2] D. Garfinkle, G. T. Horowitz, and A. Strominger, Phys. Rev. D43, 3140 (1991).

[3] J. Preskill, P. Schwarz, A. Shapere, S. Trivedi, and F. Wilczek, Mod. Phys. Lett. A6, 2353 (1991).

[4] C. F. E. Holzhey and F. Wilczek, Nucl. Phys. B380 , 447 (1992).

[5] K. Shiraishi, Phys. Lett. A166, 298 (1992).

[6] A. Papapetrou, Proc. R. Irish Acad. A51, 191 (1947)

S. D. Majumdar, Phys. Rev. 72, 930 (1947).

[7] R. C. Myers, Phys. Rev. D35, 455 (1987).

[8] J. R. Gott III and M. Alpert, Gen. Relativ. Gravit. 16, 243 (1984)

S. Giddings, J. Abbott, and K. Kuchar, Gen. Relativ. Gravit. 16, 751 (1984)

S. Deser, Jackiw, and G. 'tHooft, Ann. Phys. (NY) 152, 220 (1984)

S. Deser and R. Jackiw, ibid. 153, 405 (1984)

D. C. Duncun and E. C. Ihrig, Gen. Relativ. Gravit. 23, 381 (1991).

[9] J. R. Gott III, J. Z. Simon, and M. Alpert, Gen. Relativ. Gravit. 18, 1019 (1986).

[10] A. Staruszkiewicz, Acta Phys. Polon. 24, 734 (1963).

[11] N. S. Manton, Phys. Lett. B110, 54 (1982).

[12] R. Ward, Phys. Lett. B158, 424 (1985).

[13] M. Atiyah and N. J. Hitchin, The Geometry and Dynamics of Magnetic Monopoles (Princeton University, Princeton, NJ, 1988).

[14] P. J. Ruback, Commun. Math. Phys. 107, 93 (1986).

[15] G. W. Gibbons and P. J. Ruback, Phys. Rev. Lett. 57, 1492 (1986). 
[16] R. C. Ferrell and D. M. Eardley, Phys. Rev. Lett. 59, 1617 (1987).

[17] L. D. Landau and E. M. Lifschitz, Classical Theory of Fields, 4th ed. (Pergamon, Oxford, 1975). 